\title{
Mean-field RVB ground states of lattice models of CuNCN
}

\author{
A.L. Tchougréeff and R. Dronskowski \\ Institut für Anorganische Chemie RWTH-Aachen University, Landoltweg 1, D-52056, Aachen, Germany \\ E-mail: andrei.tchougreeff@ac.rwth-aachen.de
}

Received July 22, 2013

\begin{abstract}
Recently we proposed to describe the fascinating physics of copper carbodiimide, CuNCN, with help of the anisotropic trangular antiferromagnetic Heisenberg model with the parameters $J_{a}$ and $J_{a b}$ extending along the $a$, and $a \pm b$ lattice directions and a new frustrated Heisenberg antiferromagnetic model with exchange parameters $J_{c}, J_{a}$, and $J_{a c}$, extending along the $c, a$, and $a \pm c$ ( $c-a$-ca model) directions assuming the resonating valence bond (RVB) type of the corresponding phases. Here we discuss possible RVB ground states of these models in the mean-field approximation and show that in either case it is a two-dimensional RVB state. The difference between the models is that in the ground state of the triangular model the quasiparticle spectrum features a finite (although exponentially small) energy gap for arbitrary weak $J_{a b}$ whereas that of the $c$ - $a$-ca model shows two pseudogaps and a linear dependence of the quasiparticle density of states in the low-energy range.
\end{abstract}

PACS: 75.10.Jm Quantized spin models, including quantum spin frustration;

75.10.Kt Quantum spin liquids, valence bond phases and related phenomena;

75.50.Ee Antiferromagnetics.

Keywords: copper carbodiimide, crystal structure, spin liquid, resonating valence bond theory.

\section{Introduction}

Among the materials of the $\mathrm{MNCN}$ series $(\mathrm{M}=\mathrm{Mn}, \mathrm{Fe}$, $\mathrm{Co}, \mathrm{Ni}, \mathrm{Cu}$ ), $\mathrm{CuNCN}$ is the most bizarre [1]. In contrast to other members of the family exhibiting more or less standard antiferromagnetic behavior, $\mathrm{CuNCN}$ is a temperatureindependent (Pauli) paramagnet at room temperature and, at lower temperatures, switches to a gapped (Arrhenius) temperature dependence. Since it is not metallic in the temperature range where the Pauli paramagnetism occurs, no metal-insulator transition can be made responsible for the quasi-Arrhenius behavior either. It also does not manifest any magnetic neutron scattering [2] so there is no long-range magnetically ordered state to which one could ascribe the susceptibility decay. These findings brought us to the idea that in $\mathrm{CuNCN}$ the antiferromagnetically interacting $\mathrm{Cu}^{2+}$ local spins $1 / 2$ which are unequivocally observed in the Pauli paramagnetic phase with use of EPR may form resonating valence bond (RVB) phases $[3,4]$. This incidentally makes CuNCN an RVB material at highest temperature observed so far. The RVB hypothesis allowed us to explain the magnetic and polarized neutron experiments. Assuming that the involved exchange param- eters may also depend on the crystal geometry through respective magnetostriction terms allowed us to relate the observed anomalies in the temperature course of the $a$ and $c$ lattice parameters with transitions taking place between various RVB phases [5,6].

The above success was due to the approach we followed in Refs. 3-6. It was based on the Heisenberg model with the Hamiltonian

$$
\sum_{\mathbf{r}} \sum_{\tau} J_{\tau} \mathbf{S}_{\mathbf{r}} \mathbf{S}_{\mathbf{r}+\tau}
$$

where we went to the fermion (spinon) representation of the spins

$$
\mathbf{S}_{i}=\frac{1}{2} c_{i \alpha}^{+} \boldsymbol{\sigma}_{\alpha \beta} c_{i \beta},
$$

following the method [7] (here $c_{i \sigma}^{+}\left(c_{i \sigma}\right)$ are the fermion creation (annihilation) operators; $\boldsymbol{\sigma}_{\alpha \beta}$ are the elements of the Pauli matrices and the summation over repeating indices is assumed). We remark here that going to the spinon representation does not mean any arbitrary change of the statistics of the involved particles, since the spins in Eq. (1) themselves are the effective electron spins. They emerge from a kind of projection procedure applied to electrons 
described by, e.g., a Hubbard-like Hamiltonian. The standard technique described in Sec. 2 yielding then the quasiparticle spectra of the specific models characterized by the sets of values of the translation vector $\tau$ and the exchange parameters $J_{\boldsymbol{\tau}}$.

Formation of the RVB phases in the antiferromagnetically coupled systems is favored by frustrations which do not allow the spins to arrange in a unique long-range ordered structure $[8,9]$. In Fig. 1 we show the interactions which probably present in the CuNCN crystal as derived from structure considerations and Goodenough-Kanamori rules [10]. The magnetic behavior (the switch between the Pauli and Arrhenius regimes of the temperature course of the susceptibility) of $\mathrm{CuNCN}$ is explained by a transition between a one-dimensional (1D) and two-dimensional (2D) RVB phases occurring at a finite temperature and accompanied by opening a (pseudo)gap in the quasiparticle spectrum.

Two models describing interactions depicted in Fig. 1 and showing the required gap opening have been proposed. Originally in Refs. 3, 4 we suggested that the physics of $\mathrm{CuNCN}$ can be successfully mapped on two-dimensional anisotropic triangular lattice (ATL) model as applied to the $a b$ crystallographic planes and involving the $J_{a}$ and $J_{a b}$ interactions. It allowed us to explain the Pauli and Arrhenius regions in the temperature course of the susceptibility and anomalies in the temperature dependence of the $a$ lattice parameter. However, the hypothesis of the triangular lattice was not easy to reconcile with the intuitive picture [10] of the most important couplings which are expected to

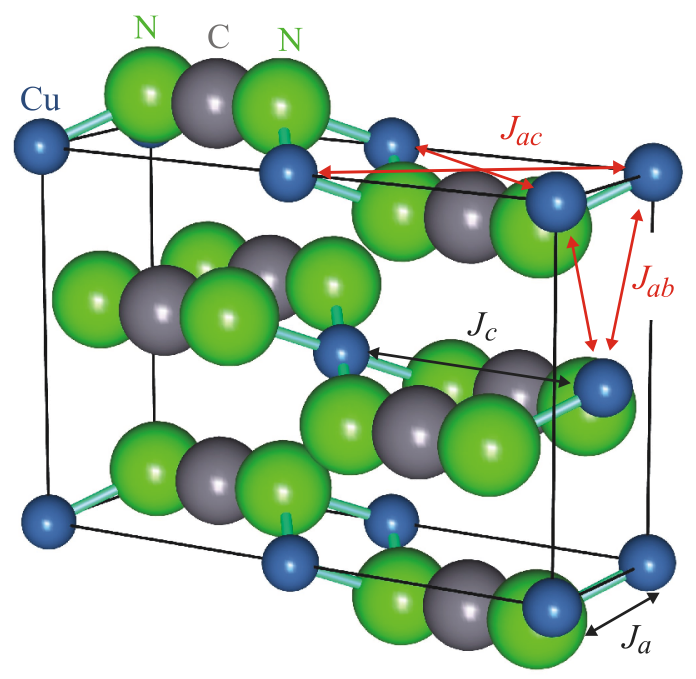

Fig. 1. (Color online) Crystal structure (unit cell) of CuNCN and exchange parameters included in the consideration. The interactions are always mediated by the $\mathrm{NCN}^{2-}$ moieties. Two stronger interactions in the $a c$ crystallographic plane $\left(J_{c}\right.$ and $\left.J_{a c}\right)$ are mediated by the $\pi$-system of $\mathrm{NCN}^{2-}$ and extend, respectively, in the $c$ and $c \pm a$ directions. Somewhat weaker $J_{a}$ contributed by a ferromagnetic counterpoise terms dependent on the hybridization at the $\mathrm{N}$ atoms extends in the $a$ direction. The weakest $J_{a b}$ as well goes through the $\mathrm{N}$ atoms. extend in the $a c$ crystallographic plane. Also the anomalies observed in the temperature course of the $c$ lattice parameter point to somewhat different model. That latter employing the $J_{c}, J_{a}$, and $J_{a c}$ interactions (c-a-ca model) has been proposed in Refs. 11, 12 and successfully reproduces even very tiny details and mutual correlations of the temperature course of the magnetic susceptibility and of the lattice parameters $a$ and $c$.

Both the ATL and the $c$ - $a$-ca models have been treated so far in the high-temperature approximation. This latter allowed us to explain the temperature dependence of the observed quantities through that of the RVB order parameters (OPs - see below). However, it remains questionable within what temperature range these predictions remain valid. At least one can be sure that the exact expressions describing the temperature course of the OPs as derived from the high-temperature expansion are wrong in the limit $T \rightarrow 0$. That is why in Refs. 11, 12 we used interpolation formulae to describe the temperature dependence of the OPs.

In the present paper in order to establish the limiting values of the OPs at the zero temperature we consider the opposite limit of two previously used models. The paper is organized as follows. In Sec. 2 we give a sketch of a route to the mean-field RVB equations and to the corresponding quasiparticle spectra and free energy expressions. Next in Sec. 3 we specify these for the two specific models (ATL and $c-a-c a$ ones) and derive and solve the self-consistency equations for the OPs relevant for the respective models at $T=0$, i.e., for their RVB ground states. Further in Sec. 4 these solutions are used to estimate the energies of the respective states and by this we derive the RVB ground state. Finally we briefly discuss the relation between the obtained zero temperature/ground state quantities and the results derived in the high-temperature approximation and give the conclusions.

\section{Equations of motion and self-consistency equations}

Equations of motion are based on the Heisenberg representation in which each operator obeys:

$$
i \hbar \dot{A}=[A, H],
$$

where $[\ldots, \ldots]$ stands for the commutator and dot for the time derivative. Applying this to the creation and annihilation operators $c_{\mathbf{r} \sigma}^{+}\left(c_{\mathbf{r} \sigma}\right)$ introduced by Eq. (2) and performing commutation, mean-field decoupling and Fourier transformation as done previously results [3] in the meanfield equations of motion:

$$
\begin{aligned}
& i \hbar \dot{c}_{\mathbf{k} \sigma}=-\frac{3}{2} \sum_{\boldsymbol{\tau}} J_{\boldsymbol{\tau}} \xi_{\boldsymbol{\tau}} \cos (\mathbf{k} \boldsymbol{\tau}) c_{\mathbf{k} \sigma}-\frac{3}{2} \sum_{\boldsymbol{\tau}} J_{\tau} \Delta_{\boldsymbol{\tau}} \cos (\mathbf{k} \boldsymbol{\tau}) c_{-\mathbf{k}-\sigma}^{+}, \\
& i \hbar \dot{c}_{\mathbf{k} \sigma}^{+}=\frac{3}{2} \sum_{\boldsymbol{\tau}} J_{\tau} \xi_{\boldsymbol{\tau}} \cos (\mathbf{k} \boldsymbol{\tau}) c_{\mathbf{k} \sigma}^{+}-\frac{3}{2} \sum_{\boldsymbol{\tau}} J_{\boldsymbol{\tau}} \Delta_{\boldsymbol{\tau}}^{*} \cos (\mathbf{k} \boldsymbol{\tau}) c_{-\mathbf{k}-\sigma},
\end{aligned}
$$


where

$$
\begin{aligned}
& \xi_{\boldsymbol{\tau}}=\left\langle c_{\mathbf{r}+\tau \sigma}^{+} c_{\mathbf{r} \sigma}\right\rangle, \\
& \Delta_{\boldsymbol{\tau}}=\left\langle c_{\mathbf{r}+\boldsymbol{\tau} \alpha} c_{\mathbf{r} \beta}\right\rangle
\end{aligned}
$$

are the order parameters (OPs - averaging over so far unknown many particle state is assumed). Equation (4) reduces to the set of $2 \times 2$ eigenvalue problems for each wave vector $\mathbf{k}$ :

$$
\left(\begin{array}{cc}
\xi_{\mathbf{k}} & \Delta_{\mathbf{k}} \\
\Delta_{\mathbf{k}}^{*} & -\xi_{\mathbf{k}}
\end{array}\right)\left(\begin{array}{l}
u_{\mathbf{k}} \\
v_{\mathbf{k}}
\end{array}\right)=E_{\mathbf{k}}\left(\begin{array}{l}
u_{\mathbf{k}} \\
v_{\mathbf{k}}
\end{array}\right)
$$

with

$$
\begin{aligned}
\xi_{\mathbf{k}} & =-3 \sum_{\boldsymbol{\tau}} J_{\tau} \xi_{\tau} \cos (\mathbf{k} \boldsymbol{\tau}), \\
\Delta_{\mathbf{k}} & =3 \sum_{\boldsymbol{\tau}} J_{\tau} \Delta_{\boldsymbol{\tau}} \cos (\mathbf{k} \boldsymbol{\tau}),
\end{aligned}
$$

and the eigenvalues

$$
E_{\mathbf{k}}= \pm \sqrt{\xi_{\mathbf{k}}^{2}+\left|\Delta_{\mathbf{k}}\right|^{2}}
$$

This set of equations formally closes by the self-consistency conditions:

$$
\begin{aligned}
& \xi_{\boldsymbol{\tau}}=-\frac{1}{2 N} \sum_{\mathbf{k}} \exp (i \mathbf{k} \boldsymbol{\tau}) \frac{\xi_{\mathbf{k}}}{E_{\mathbf{k}}} \tanh \left(\frac{E_{\mathbf{k}}}{2 \theta}\right), \\
& \Delta_{\boldsymbol{\tau}}=\frac{1}{2 N} \sum_{\mathbf{k}} \exp (-i \mathbf{k} \boldsymbol{\tau}) \frac{\Delta_{\mathbf{k}}}{E_{\mathbf{k}}} \tanh \left(\frac{E_{\mathbf{k}}}{2 \theta}\right)
\end{aligned}
$$

for the OPs. We, however, follow somewhat different, but equivalent method. For the complex OPs we introduce a polar representation:

$$
\Delta_{\tau}=\eta_{\tau} \mathrm{e}^{i \varphi_{\tau}}
$$

The standard moves foreseen to account for the $S U(2)$ symmetry of the solutions allow one to exclude the cross terms in OPs from the above expression under the square root which leads to the systems of conditions

$$
\xi_{\tau} \xi_{\tau}^{\prime}=-\eta_{\tau} \eta_{\tau}^{\prime} \cos \left(\varphi_{\tau}-\varphi_{\tau}^{\prime}\right)
$$

which can be satisfied for our specific models. The $S U(2)$ symmetry thus allows to express the free energy for either of the so far used models as

$$
F=\frac{3}{2} \sum_{\boldsymbol{\tau}} J_{\tau} \zeta_{\tau}^{2}-\frac{2 \theta}{|B Z|} \int_{B Z} \ln \left(2 \cosh \left(\frac{E_{\mathbf{k}}}{2 \theta}\right)\right) d^{2} \mathbf{k},
$$

where $\theta=k_{B} T$ and the effective OPs are introduced $\zeta_{\tau}=$ $=\sqrt{\xi_{\tau}^{2}+\eta_{\tau}^{2}}, B Z$ stands for the integration over the Brillouin zone, and $|B Z|$ stands for their areas specific for each model. The auxiliary bosons assuring the approximate projection to the single occupancy subspace in case of the unity filling (zero hole concentration) are decoupled from other averages $\left(\xi_{\tau}, \eta_{\tau}\right.$, and/or $\left.\zeta_{\tau}\right)$ entering the free energy expression (8) [7] and thus can be omitted.

It easy to check that for $\theta \rightarrow 0$ the "kinetic" energy contribution transforms into the integral over the $\mathrm{BZ}$ from the quasiparticle spectrum itself:

$$
\begin{aligned}
& T=-\frac{2 \theta}{|B Z|} \int_{B Z} \ln \left(2 \cosh \left(\frac{E_{\mathbf{k}}}{2 \theta}\right)\right) d^{2} \mathbf{k} \rightarrow \\
& \rightarrow-\frac{1}{|B Z|} \int_{B Z} E_{\mathbf{k}} d^{2} \mathbf{k}=-\int g(\varepsilon) \varepsilon d \varepsilon,
\end{aligned}
$$

where $g(\varepsilon)$ stands for the quasiparticle density of states (qDoS). It is obviously uniform of the first power with respect to $\zeta_{\tau}$ 's in either case. The "kinetic" energy contribution (9) complemented by the "potential" energy contribution quadratic with respect to the OPs (the first term in Eq. (8) which thus yields the ground-state energy as a function of effective OPs). The allowed states of the system are the minima of the ground-state energy with respect to OPs. These are an equivalent alternative to solving the self-consistency conditions (5) which better suits our purpose.

\section{Quasiparticle dispersion laws of the specific frustrated models}

As we mentioned in the Introduction two frustrated antiferromagnetic Heisenberg models have been considered in relation to CuNCN. Hereinafter we shall consider these models in sequel.

\subsection{ATL model}

This model is apparently the simplest one where frustration can be observed and thus it has been a subject of numerous studies by a variety of methods. They all quite consistently indicate towards existence of spin-liquid (RVB) states in certain regions of the parameter space. It is obvious that at very small values of the frustrating interaction $J_{a}$ the system occurs in an antiferromagnetic ground state of a square lattice with the single parameter $J_{a b}$. Numerical study of the opposite limit $\left(J_{a b}<J_{a}\right)$ which is more relevant for the CuNCN physics in the mean-field RVB approximation showed [13] that the 1D- and 2D-RVB phases are possible. Formally the ATL model is characterized by the translation vectors $\pm \boldsymbol{\tau}$ with $\boldsymbol{\tau}$ taking three values in the $a b$ crystallographic plane $\tau_{i} ; i=1-3$;

$$
\tau_{1}=(1,0) ; \tau_{2}=\left(\frac{1}{2}, \frac{\sqrt{3}}{2}\right) ; \tau_{3}=\left(\frac{1}{2},-\frac{\sqrt{3}}{2}\right)
$$

with the interaction of the strength $J_{a}$ along the lattice vector $\tau_{1}$ (two neighbors) and with a somewhat smaller strength $J_{a b}$ along the lattice vectors $\tau_{2}$ and $\tau_{3}$ (two neighbors along each). This setting results in the dispersion law

$E_{\mathbf{k}}^{2}=9\left(J_{a}^{2} \zeta_{a}^{2} \cos ^{2} x+J_{a b}^{2} \zeta_{a b}^{2}+J_{a b}^{2} \zeta_{a b}^{2} \cos x \cos \sqrt{3} y\right)$

where we set $x$ for $\mathbf{k}_{x}$ and $y$ for $\mathbf{k}_{y}$ for brevity. Two possible nontrivial regimes correspond to the $1 \mathrm{D}-\mathrm{RVB}$ 

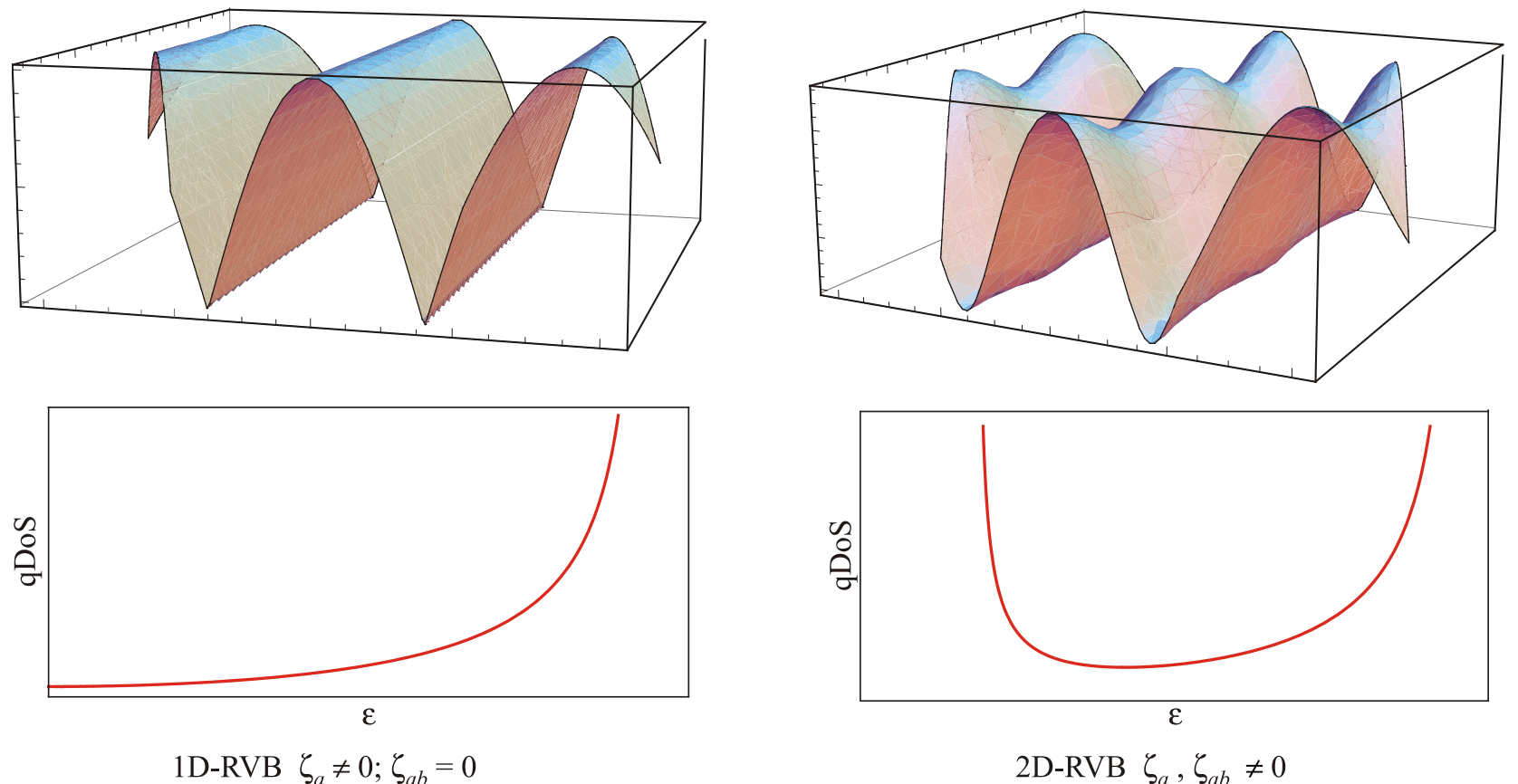

1D-RVB $\zeta_{a} \neq 0 ; \zeta_{a b}=0$

2D-RVB $\zeta_{a}, \zeta_{a b} \neq 0$

Fig. 2. (Color online) Dispersion laws (quasiparticle energy — applicate, vs 2D-wave vector in the Brillouin zone — abscissa and ordinate) of the ATL-RVB model for characteristic values of the OPs $\zeta_{a}$ and $\zeta_{a b}$ indicating key features of the quasiparticle spectrum in respective RVB states and the sketches of the relevant qDoS (see text for the details).

with $\zeta_{a} \neq 0 ; \zeta_{a b}=0$ and the 2D-RVB with $\zeta_{a}, \zeta_{a b} \neq 0$. Corresponding quasiparticle dispersion laws are shown in Fig. 2.

In the 1D-RVB state the quasiparticle's dispersion vanishes in the $y$ direction:

$$
E_{\mathbf{k}}=3 J_{a} \zeta_{a}|\cos x|
$$

The spectrum is gapless along the nodal lines $\mathbf{k}_{x}= \pm \pi / 2$ and manifests ridges extended in the $y$ direction at $\mathbf{k}_{x}=0, \pm \pi$. The corresponding $\mathrm{qDoS}$ :

$$
g(\varepsilon)=\frac{2}{\pi \sqrt{9 J_{a}^{2} \zeta_{a}^{2}-\varepsilon^{2}}}
$$

is depicted in Fig. 2. It diverges on the upper bound of the spectrum. The low-energy/low-temperature behavior of the system in the 1D-RVB state is controlled by a constant qDoS at the zero energy, which perfectly maps to the temperature-independent paramagnetic susceptibility in the corresponding phase.

As one can easily see the most characteristic difference between the 1D- and 2D-RVB states of the ATL model is the gap in the quasiparticle spectrum consequently reflected in the form of the qDoS schematically shown in the same figure. Although the quasiparticles acquire a dispersion in the $y$ direction and both the nodal lines $\mathbf{k}_{x}= \pm \pi / 2$ and the ridges $\mathbf{k}_{x}=0, \pm \pi$ acquire a goffer (see Fig. 2) one can see from the figure and Table 1 that in either case the amount of dispersion in the $y$ direction is of the higher order of magnitude as compared to other characteristic energies.
Table 1. Critical points of the quasiparticle spectrum in the gapped spin-liquid 2D-RVB state of ATL model. $\left(\mathbf{k}_{x}, \mathbf{k}_{y}\right)$ stands for the coordinates of the critical point in the Brillouin zone, $n$ is the degeneracy - total number of points of the given type; other entries are self-explanatory

\begin{tabular}{c|c|c|c}
\hline$\left(\mathbf{k}_{x}, \mathbf{k} y\right)$ & $n$ & $E^{2}$ & Point type \\
\hline$\left(0, \frac{\pi l}{\sqrt{3}}\right) ; l=-2,0,2$ & 3 & $9\left(J_{a}^{2} \zeta_{a}^{2}+2 J_{a b}^{2} \zeta_{a b}^{2}\right)$ & maximum \\
\hline$\left( \pm \pi, \pm \frac{\pi}{\sqrt{3}}\right)$ & 4 & $9\left(J_{a}^{2} \zeta_{a}^{2}+2 J_{a b}^{2} \zeta_{a b}^{2}\right)$ & maximum \\
\hline$\left( \pm \frac{\pi}{\sqrt{3}}\right)$ & 2 & $9 J_{a}^{2} \zeta_{a}^{2}$ & saddle \\
\hline$( \pm \pi, 0)$ & 2 & $9 J_{a}^{2} \zeta_{a}^{2}$ & saddle \\
\hline$\left( \pm \frac{\pi}{2}, \pm \frac{\pi \sqrt{3}}{2}\right)$ & 4 & $9 J_{a b}^{2} \zeta_{a b}^{2}$ & saddle \\
\hline$\left( \pm \arccos \left(-\frac{J_{a b}^{2} \zeta_{a b}^{2}}{2 J_{a}^{2} \zeta_{a}^{2}}\right), \pm \frac{2 \pi}{\sqrt{3}}\right)$ & 4 & $9 J_{a b}^{2} \zeta_{a b}^{2}\left(1+\frac{3}{4} \frac{J_{a b}^{2} \zeta_{a b}^{2}}{J_{a}^{2} \zeta_{a}^{2}}\right)$ & saddle \\
\hline$\left( \pm \arccos \left(\frac{J_{a b}^{2} \zeta_{a b}^{2}}{2 J_{a}^{2} \zeta_{a}^{2}}\right), \pm \frac{\pi}{\sqrt{3}}\right)$ & 4 & $9 J_{a b}^{2} \zeta_{a b}^{2}\left(1-\frac{1}{4} \frac{J_{a b}^{2} \zeta_{a b}^{2}}{J_{a}^{2} \zeta_{a}^{2}}\right)$ & minimum \\
\hline \hline$\left.\left(-\frac{J_{a b}^{2} \zeta_{a b}^{2}}{2 J_{a}^{2} \zeta_{a}^{2}}\right), 0\right)$ & 2 & $9 J_{a b}^{2} \zeta_{a b}^{2}\left(1-\frac{1}{4} \frac{J_{a b}^{2} \zeta_{a b}^{2}}{J_{a}^{2} \zeta_{a}^{2}}\right)$ & minimum \\
\hline \hline
\end{tabular}


Specifically in the low-energy range the splitting between the saddle points of the quasiparticle spectrum are of the fourth order with respect to presumably small OP $\zeta_{a b}$ whereas the energy gap itself is of the second order. This brings us to the idea that neglecting the $y$ dispersion when calculating the integral characteristics of the system does not affect the precision catastrophically. This may be considered as a quasi-one-dimensional approximation for the spectrum, which then takes the form

$$
E_{\mathbf{k}}=3 \sqrt{J_{a}^{2} \zeta_{a}^{2} \cos ^{2} x+J_{a b}^{2} \zeta_{a b}^{2}}
$$

Inserting this in the standard definition of the qDoS we obtain

$$
g(\varepsilon)=\frac{2 \varepsilon}{\pi \sqrt{\left(9 J_{a}^{2} \zeta_{a}^{2}+9 J_{a b}^{2} \zeta_{a b}^{2}-\varepsilon^{2}\right)\left(\varepsilon^{2}-9 J_{a b}^{2} \zeta_{a b}^{2}\right)}}
$$

for the quasiparticles in a band ranging from $3 J_{a b} \zeta_{a b}$ to $3 \sqrt{J_{a}^{2} \zeta_{a}^{2}+J_{a b}^{2} \zeta_{a b}^{2}}$ with its lower boundary being as explained above only slightly higher than the lower boundary of the exact spectrum and with the upper boundary being located between the exact upper boundary and its logarithmic peak. Of course, the precise form of the van Hove singularities is not reproduced: instead of logarithmic divergency we have the power-like one, but as we hope (see above) it does not fatally affect the integral values.

With use of the qDoS (13) one can easily write the explicit expression for the ground-state energy of the 2D-RVB state as relying on the general expression (8) where the integral is done analytically [14]:

$$
F(\theta=0)=3 J_{a} \zeta_{a}^{2}+6 J_{a b} \zeta_{a b}^{2}-\frac{6}{\pi} \sqrt{J_{a}^{2} \zeta_{a}^{2}+J_{a b}^{2} \zeta_{a b}^{2}} \mathrm{E}(k)
$$

Here $\mathrm{E}(k)$ is the complete elliptic integral of the 2nd kind of the modulus $k$ given by

$$
k^{2}=\frac{J_{a}^{2} \zeta_{a}^{2}}{J_{a}^{2} \zeta_{a}^{2}+J_{a b}^{2} \zeta_{a b}^{2}}
$$

This result is not unexpected since it has a form characteristic for one-dimensional systems [15]. Taking derivatives with respect to the OPs $\zeta_{a b}, \zeta_{a}$ and setting the former equal to zero results in the self-consistency equations

$$
\begin{aligned}
& 1=\frac{J_{a}}{\pi \sqrt{J_{a}^{2} \zeta_{a}^{2}+J_{a b}^{2} \zeta_{a b}^{2}}}(\mathrm{~K}(k)-\mathrm{D}(k)), \\
& 1=\frac{J_{a b}}{2 \pi \sqrt{J_{a}^{2} \zeta_{a}^{2}+J_{a b}^{2} \zeta_{a b}^{2}}} \mathrm{~K}(k)
\end{aligned}
$$

( $\mathrm{K}$ is the complete elliptic integral of the first kind, $\mathrm{D}=(\mathrm{K}-\mathrm{E}) / k^{2}$ is the auxillary elliptic integral defined in [14]), which are remarkably similar to the self-consistency conditions in the one-dimensional Hubbard problem as written in Ref. 17, the first being one for the bond order and the second being analogous to that for the gap or mag- netization with $J_{a b}$ taking part of the interaction parameter of the Hubbard model and $3 J_{a} \zeta_{a}$ being the effective one-dimensional bandwidth. In the 1D-RVB state the first of the conditions (15) yields the amplitude of the OP $\zeta_{a}$ reached at the zero temperature:

$$
\zeta_{a}=1 / \pi
$$

which is in perfect agreement with the numerical result of [16] (and equals to the bond order on the one-dimensional chain without interaction). Inserting this in the second of the two conditions (15), neglecting the terms containing $\zeta_{a b}$ as compared to those with $\zeta_{a}$ in the sums, and using the logarithmic asymptotic of $\mathrm{K}$ we arrive to the estimate for $\zeta_{a b}$ and for the gap in the 2D-RVB state:

$$
\begin{gathered}
\zeta_{a b}=\frac{4}{\pi} \frac{J_{a}}{J_{a b}} \exp \left(-\frac{2 J_{a}}{J_{a b}}\right), \\
3 J_{a b} \zeta_{a b}=\frac{12 J_{a}}{\pi} \exp \left(-\frac{2 J_{a}}{J_{a b}}\right)
\end{gathered}
$$

at zero temperature. The latter result is in a fair agreement with the numerical study [18] where the pre-exponential factor in the gap was estimated to be 3.50 as compared to our $12 / \pi \approx 3.82$. Although the factor in the exponent was estimated to be 1.61 in [18] against our estimate of two, the general form of the dependence of the characteristics of the model on its parameters is reproduced. One can, however, expect that neglecting the dispersion in the $y$ direction may well affect precisely the numerical coefficients of that kind. These results show that at the zero temperature some nonvanishing value of the $\zeta_{a b}$ OP and the energy gap appear at arbitrary weak interaction $J_{a b}$ so that no critical point with respect to the anisotropy $J_{a b} / J_{a}$ should be expected in variance with the numerical result obtained on a finite although large chunk of the lattice [16,18]. One has to give preference to the above approximate analytic result over a numerical one in this case since one can imagine that detecting an exponentially small gap or OP in a numerical experiment may be problematic.

A further move consists in using the above expression for $\zeta_{a b}$ for further iteration. Retaining the terms proportional to $\Lambda k^{\prime 2} ; \Lambda=\ln 4 / k^{\prime}, k^{\prime 2}=1-k^{2}$ in the equation for $\zeta_{a}$ so that $\mathrm{K}(k)-\mathrm{D}(k) \approx 1-\Lambda k^{\prime 2} / 2$ and keeping the terms up to second order in $\zeta_{a b}$ we obtain

$$
\zeta_{a}=\frac{1}{\pi}-\frac{\pi J_{a b}}{J_{a}} \zeta_{a b}^{2}=\frac{1}{\pi}-\frac{16}{\pi} \frac{J_{a}}{J_{a b}} \exp \left(-\frac{4 J_{a}}{J_{a b}}\right),
$$

which represents the estimate of the "bond order" variation in the 2D-RVB state as compared to the 1D-RVB state. This finding is in a fair and remarkable agreement with the numerical result of [18] where it was shown that in the region where the 2D-RVB state develops $\left(\zeta_{a b} \neq 0\right)$ the $\zeta_{a}$ OP manifests a very weak depletion as compared to its 1D-RVB $\left(\zeta_{a b}=0\right)$ value. 


\section{2. $c$-a-ca model}

As we mentioned in the Introduction the ATL model is not fully consistent when it comes to interpretation of the low-temperature anomalies in the temperature course of the $c$ lattice constant $[6,11,12]$. Thus more antiferromagnetic couplings acting along translations $\boldsymbol{\tau}_{i} ; i=1-4$; $\boldsymbol{\tau}_{1}=(a, 0) ; \quad \tau_{2}=(0, c) ; \quad \tau_{3}=(a, c) ; \quad \tau_{4}=(a,-c)$ with the interaction of the strength $J_{a}$ along $\tau_{1}$ (two neighbors), with a strength $J_{c}$ along $\tau_{2}$ (two neighbors as well), and interaction of the strength $J_{a c}$ along $\tau_{3}$ and $\tau_{4}$ (two neighbors along each) — see Fig. 1 - have been proposed. Either interactions along $\boldsymbol{\tau}_{1}$ and $\boldsymbol{\tau}_{2}$ or those along $\boldsymbol{\tau}_{3}$ and $\boldsymbol{\tau}_{4}$ taken separately must lead to an antiferromagnetic state. However, when considered simultaneously they interfere leading to the required frustration not allowing the spins to arrange in any magnetically ordered state. This model generalizes the known Nersesyan-Tsvelik model [19] which derives from ours by setting $J_{a}=J_{c}$ which is clearly not the case for CuNCN. Nevertheless, it is known [19] that the RVB state of the Nersesyan-Tsvelik model is stable with respect to formation of an antiferromagnetic phase. For similar systems a variety of RVB states have been proposed $[7,13]$. Ground states of a similar, but spatially isotropic $J_{1} J_{2} J_{3}$ model have been treated recently by various methods, and it has been shown that spin-liquid states are very probable [20].

3.2.1. Dispersion law and ground-state energy. Applying the moves described in Sec. 2 one can select the relative phases $\varphi_{\tau}$ in Eq. (6) for the complex OPs so that the Eq. (7) are satisfied and to arrive to the spectrum of quasiparticles of the form [12]

$E_{\mathbf{k}}^{2}=9\left(J_{a}^{2} \zeta_{a}^{2} \cos ^{2} x+J_{c}^{2} \zeta_{c}^{2} \cos ^{2} z+4 J_{a c}^{2} \zeta_{a c}^{2} \cos ^{2} x \cos ^{2} z\right)$,

where we set $x=\mathbf{k}_{x} ; z=\mathbf{k}_{z}$ and the effective OPs $\zeta_{a}, \zeta_{c}$, $\zeta_{a c}$ describe the states of the model.
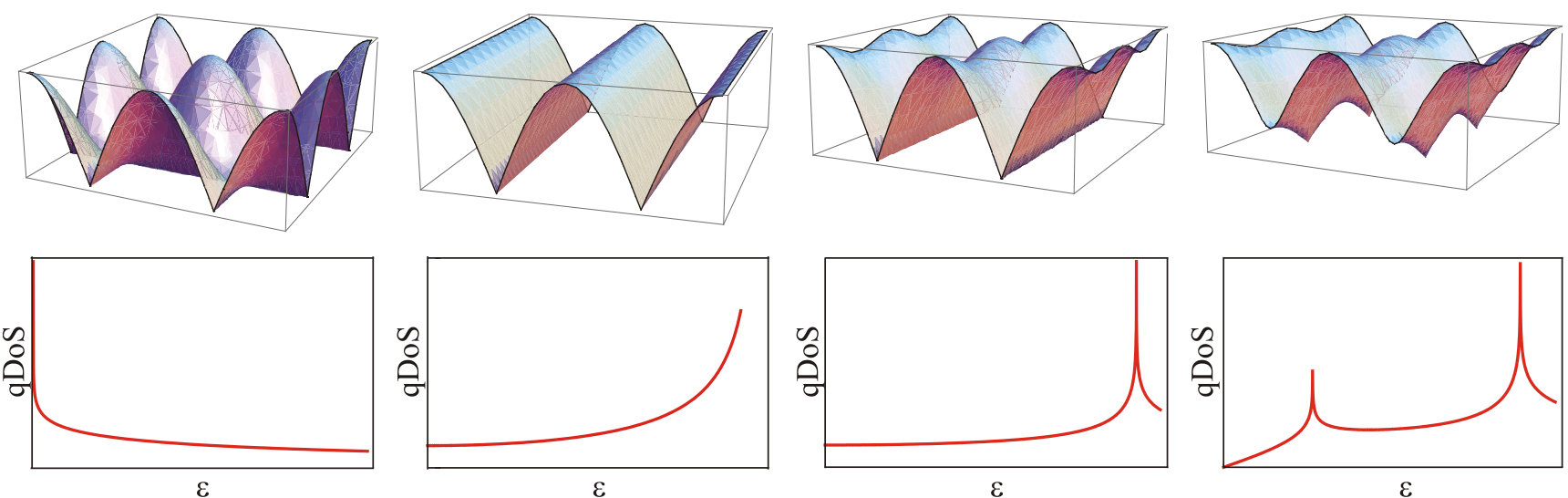

2D-RVB $\zeta_{a}, \zeta_{c}=0 ; \zeta_{a c} \neq 0$

Obviously the $c$ - $a$ - $c a$ model is much richer than the ATL one. Its versatile quasiparticle spectrum (18) is represented in Fig. 3 originally published in Ref. 12. It shows three principal regimes (each eventually realizable by various combinations of the effective OPs): (i) one with two pairs of lines of nodes (gapless 2D-RVB), (ii) one with a pair of lines of nodes (termed as 1D- and Q1D-RVB states), (iii) and one with two pseudogaps and four nodal points (pseudogapped 2D-RVB).

If either of the OPs $\zeta_{a}$ or $\zeta_{c}$ is the only nonvanishing $\mathrm{OP}$, the dispersion law acquires corresponding lines of nodes $z= \pm \pi / 2$ (or $x= \pm \pi / 2$ ) where the quasiparticles have zero energy. These states can be unequivocally characterized as 1D-RVB ones since the dispersion of quasiparticles occurs in only one crystallographic direction ( $a$ or $c$ ). The qDoS in the 1D-RVB states of the $c-a-c a$ model follows the pattern known from the ATL model. It is constant at zero energy, but shows a power-like divergency at the ceiling of the quasiparticle band due to the dispersionless ridge in the dispersion law [3], like in the ATL model [18].

If both OPs $\zeta_{a, c}$ vanish and the OP $\zeta_{a c}$ does not, two pairs of nodal lines exist along which the quasiparticles have zero energy. In this state the qDoS diverges logarithmically at zero energy. Since the quasiparticles have dispersion in both directions in the Brillouin zone this state has to be characterized as a (gapless) 2D-RVB state.

If either of the nonvanishing OPs $\zeta_{a, c}$ is complemented by the nonvanishing OP $\zeta_{a c}$, quasi-1D-RVB (Q1D-RVB) states appear (not to be mixed with the above quasi-one-dimensional approximation (12)-(15) as applied to treat true 2D-RVB states of the ATL model). The difference with the true 1D-RVB states is that in the Q1D-RVB states one finds a nonvanishing dispersion along the node lines, so that their dispersion law have local maxima and saddle points instead of the ridge characteristic for the true 1D-RVB

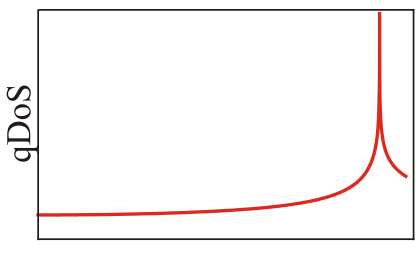

$\varepsilon$

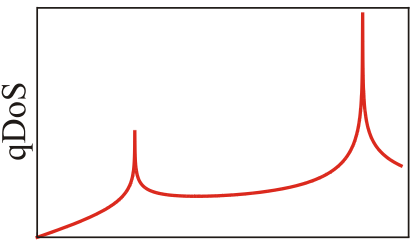

$\varepsilon$

2D-RVB $\zeta_{c}, \zeta_{a} \neq 0$

Fig. 3. (Color online) Dispersion laws (quasiparticle energy — applicate, vs $2 \mathrm{D}$-wave vector in the Brillouin zone $\mathbf{k} \in[-\pi, \pi] \times[-\pi, \pi]$ - abscissa and ordinate) of the $c-a-c a$-RVB model for several characteristic values of the OPs $\zeta_{a}$, $\zeta_{c}$, and $\zeta_{a c}$ indicating key features of the quasiparticle spectrum in different RVB states and the sketches of the relevant qDoS (normalized number of states) vs quasiparticle energy $\varepsilon$ (see text for the details). 
state. Thus, the qDoS develops a finite hop at the ceiling of the quasiparticle band and a logarithmic singularity at a pseudogap which is a saddle point of the quasiparticle dispersion law. Since at the zero energy the quasiparticle spectrum is formed by acoustic spinons propagating in one direction and only rudimentary dispersed in another one considering these states as quasi-one-dimensional is well based.

By contrast, if the nonvanishing OP's are $\zeta_{c}$ and $\zeta_{a}$, then (irrespective of the OP $\zeta_{a c}$ ) there are no lines of nodes, but four nodal points $(\mathbf{k}=( \pm \pi / 2, \pm \pi / 2))$ where the quasiparticle energies vanish. The two possible states of this type are thus pseudogapped 2D-RVB states. The qDoS in 2D-RVB states vanishes at the zero energy, being proportional to the energy well below the smaller pseudogap. Otherwise the quasiparticle dispersion law has saddle points at the two pseudogap energies and, thus, the qDoS of $2 \mathrm{D}-\mathrm{RVB}$ state develops two logarithmic singularities. In the NersesyanTsvelik model $\left(J_{a}=J_{c}\right)$ two logarithmic peaks coalesce and only one pseudogap manifests. If all three OPs are nonvanishing this does not significantly affect the character of the quasiparticle spectrum. As in the previous pseudogapped 2D-RVB state this one contains four nodal points and the only difference is somewhat larger width of the quasiparticle band. Characteristic feature of the pseudogapped state - the linear course of the qDoS at low energy survives, however, the split of the OP $\zeta_{a c}$ from zero.

The specific OP values for the ground states of the $c-a-c a$ model derive from its ground-state energy which can be written immediately [7] as

$$
F=3 J_{a} \zeta_{a}^{2}+3 J_{c} \zeta_{c}^{2}+6 J_{a c} \zeta_{a c}^{2}-\frac{1}{4 \pi^{2}} \int_{B Z} E_{\mathbf{k}} d^{2} \mathbf{k} .
$$

Possible solutions of the minima conditions of (19) with respect to possible combinations of OPs are considered hereinafter.

3.2.2. Self-consistency equations for the OPs and their solutions. Like in the ATL model both the "kinetic" and "potential" energy terms in Eq. (19) are function where the OPs enter as quadratic expressions. Thus taking derivatives with respect to the OPs always results in self-consistency equations allowing for solutions where all or at least some of the OPs vanish. Clearly, having one or more of the OPs vanishing significantly simplifies the situation and makes analytical solution possible at least in certain cases. In the present section we shall consider possible solutions in a sequel.

Pseudogapless 2D-RVB state. This state is characterized by the single nonvanishing OP $\zeta_{a c} \neq 0$. The quasiparticle spectrum (18) simplifies considerably:

$$
E_{\mathbf{k}}=6 J_{a c} \zeta_{a c}|\cos x \cos z|,
$$

and the integration over the BZ can be performed. Combining its result with the potential energy we obtain

$$
F=-\frac{24 J_{a c} \zeta_{a c}}{\pi^{2}}+6 J_{a c} \zeta_{a c}^{2},
$$

which after minimizing with respect to $\zeta_{a c}$ yields

$$
\zeta_{a c}=2 / \pi^{2}
$$

as the self consistent value.

One-dimensional RVB states. These appear when only one of either OPs $\zeta_{a}$ or $\zeta_{c}$ is nonvanishing, whereas $\zeta_{a c}=0$. This leads to the quasiparticle spectrum of the form

$$
E_{\mathbf{k}}=3 J_{\tau} \zeta_{\tau}\left|\cos \mathbf{k}_{\tau}\right|
$$

$(\tau=a, c)$ which is already known from the 1D-RVB state of the ATL model (see $[16,18]$ and above). Then we simply write

$$
\zeta_{a, c}=1 / \pi,
$$

which do take place separately for $\tau=a$ and $\tau=c$.

Quasi-one-dimensional RVB states. Such states correspond to the situations when the OP $\zeta_{a c}$ and only one of the OPs $\zeta_{a}$ or $\zeta_{c}$ are nonvanishing. In this case the quasiparticle spectrum acquires the form (for definiteness we assume $\zeta_{a} \neq 0$ ):

$$
E_{\mathbf{k}}=3 J_{a} \zeta_{a}|\cos x| \sqrt{1+A^{2} \cos ^{2} z},
$$

where

$$
A=\frac{2 J_{a c} \zeta_{a c}}{J_{a} \zeta_{a}}
$$

Due to $J_{a c}$ interactions the dispersion appears in the $z$ direction. Nevertheless, the integration within the $\mathrm{BZ}$ factorizes and as previously can be performed analytically yielding:

$$
-\frac{12}{\pi^{2}} \sqrt{J_{a}^{2} \zeta_{a}^{2}+4 J_{a c}^{2} \zeta_{a c}^{2}} \mathrm{E}(k) ; k^{2}=\frac{4 J_{a c}^{2} \zeta_{a c}^{2}}{J_{a}^{2} \zeta_{a}^{2}+4 J_{a c}^{2} \zeta_{a c}^{2}}
$$

which is the result fairly well known in the mean-field theory of one-dimensional systems [15] and in various contexts appeared in our work $[5,17]$. It coincides with that for the one-dimensional Hubbard model for electrons with onsite repulsion [17]. The parameters of the respective models enter the answer Eq. (23) unsymmetrically: one of the parameters is responsible for the width of the quasiparticle band whereas another one for possible gap-like features in the quasiparticle spectrum. In the Hubbard model these were, respectively, intersite electron hopping parameter and the on-site electron-electron repulsion parameter. In its turn in our quasi-one-dimensional approximation for the ATL model [5] (see above) the bandwidth-like parameter $J_{a}$ was the exchange parameter along the chains whereas the oblique exchange parameter $J_{a b}$ took the part of the effective interaction. In the present model the band-width (effective hopping) parameter is that of the exchange along the structural diagonal $\left(J_{a c}\right)$ whereas the exchange along 
the unit cell edges $\left(J_{a}\right.$ or alternatively $\left.J_{c}\right)$ takes part of the effective interaction.

Further moves are obvious. The "kinetic" energy (23) has to be differentiated with respect to the OPs $\zeta_{a c}$ and $\zeta_{a}$. Taking the derivative with respect to $\zeta_{a}$ yields

$$
-\frac{12}{\pi^{2}} \frac{J_{a}^{2} \zeta_{a} \mathrm{~K}(k)}{\sqrt{J_{a}^{2} \zeta_{a}^{2}+4 J_{a c}^{2} \zeta_{a c}^{2}}}
$$

the value of the modulus $k$ is given by Eq. (23). Combining this with the corresponding derivative of the potential energy and excluding the trivial solution $\zeta_{a}=0$ we arrive to the self-consistency equation

$$
1=\frac{2}{\pi^{2}} \frac{J_{a}}{\sqrt{J_{a}^{2} \zeta_{a}^{2}+4 J_{a c}^{2} \zeta_{a c}^{2}}} \mathrm{~K}(k)
$$

for the OP $\zeta_{a}$. The logarithmic singularity of the elliptic integral $\mathrm{K}(k)$ at $k=1$ guarantees as usual the existence of a nontrivial solution and the corresponding pseudogap in the quasiparticle spectrum for an arbitrary weak "effective interaction" $J_{a}$.

Analogous move with respect to the "bandwidth parameter" $\zeta_{a c}$ yields (after excluding the trivial solution for the OP $\zeta_{a c}$ ) the self-consistency equation for the latter:

$$
1=\frac{4}{\pi^{2}} \frac{J_{a c}}{\sqrt{J_{a}^{2} \zeta_{a}^{2}+4 J_{a c}^{2} \zeta_{a c}^{2}}}(\mathrm{~K}-\mathrm{D}) .
$$

The equation is fairly analogous to equations for the "bond orders" of Refs. 5, 17 and the ATL model. The solution in the limit of $\zeta_{a} \rightarrow 0$ is consistently $\zeta_{a c}=2 / \pi^{2}$.

Iterating the last solution we obtain for the small values of $\zeta_{a}$

$$
\zeta_{a}=\frac{16 J_{a c}}{\pi^{2} J_{a}} \exp \left(-\frac{2 J_{a c}}{J_{a}}\right)
$$

and analogous expression for $\zeta_{c}$. This indicates that the pseudogap is exponentially small for small interaction parameters $J_{\tau} ; \tau=a, c$.

Iterating the equation for the OP $\zeta_{a c}$ we obtain

$$
\zeta_{a c}^{2}=\frac{4}{\pi^{4}}(\mathrm{~K}-\mathrm{D})^{2}-\frac{J_{a}^{2} \zeta_{a}^{2}}{4 J_{a c}^{2}}
$$

which shows a quadratic dependence of $\zeta_{a c}$ on the OP $\zeta_{a}$ $\left(\zeta_{c}\right)$ as that latter splits from zero.

More detailed study is based on the expansion $\mathrm{K}(k)-\mathrm{D}(k) \approx 1-(\Lambda-3 / 2) k^{\prime 2} / 2$ where one cannot consider $3 / 2$ in the multiplier at the squared complementary modulus $k^{\prime}$ to be small as compared to the logarithmically divergent term $\Lambda$ since that latter has as its argument exponentially small complementary modulus itself so that $\Lambda$ and $3 / 2$ are in fact of the same order of magnitude. Thus employing Eq. (26) we get

$$
k^{\prime}=4 \exp \left(-\frac{2 J_{a c}}{J_{a}}\right) ; \Lambda=\frac{2 J_{a c}}{J_{a}},
$$

and we finally arrive to

$$
\zeta_{a c}^{2}=\frac{4}{\pi^{4}}\left[1+16\left(\frac{1}{2}-\frac{2 J_{a c}}{J_{a}}\right) \exp \left(-\frac{4 J_{a c}}{J_{a}}\right)\right]
$$

which shows that the band width OP acquires an exponentially small correction as well.

The opposite limit is as well of interest. The selfconsistency condition (24) for the OP $\zeta_{a}$ yields correct solution for the case $\zeta_{a c}=0$ which is the solution for the 1D-RVB state $\left(\zeta_{a}=1 / \pi\right)$. This corresponds to the values of the modulus $k$ of the elliptic integrals close to zero. In that limit we use an existence condition produced by equating the right hand sides of two self-consistency Eqs. (24), (25):

$$
J_{a} \mathrm{~K}=2 J_{a c}(\mathrm{~K}-\mathrm{D})
$$

It determines a self-consistent value of the modulus $k$, which can be found only for the parameters' range

$$
0 \leq J_{a} \leq J_{a c}
$$

since for the elliptic integrals the following holds:

$$
0 \leq \frac{\mathrm{K}-\mathrm{D}}{\mathrm{K}} \leq \frac{1}{2}
$$

That means that in the parameters range where $J_{a c}$ is too small as compared to $J_{a}$ (or $J_{c}$ ) the Q1D-RVB states (respectively, with $\zeta_{a}, \zeta_{a c} \neq 0$ or $\zeta_{c}, \zeta_{a c} \neq 0$ ) do not exist whereas such a state always exists for $J_{a, c}$ small as compared to $J_{a c}$. This result is very remarkable since it exactly reproduces respective estimate obtained with use of the high-temperature expansion. That latter places the boundaries between the 1D- and Q1D-RVB phases $\left(\zeta_{\tau} \neq 0\right.$, $\zeta_{a c}=0$ and $\zeta_{\tau}, \zeta_{a c} \neq 0, \tau=a$ or $c$ ) right at $J_{\tau}=J_{a c}$.

Approximate solutions can be found in the limit of small $\zeta_{a c}$. In this case the elliptic integrals expand in power series with respect to $k^{2}$ [14] so that one easily obtains for the self-consistent value of $k^{2}$ in the lowest order:

$$
\frac{k^{2}}{4}=\frac{2\left(J_{a c}-J_{a}\right)}{2 J_{a}-J_{a c}}=\frac{J_{a c}^{2} \zeta_{a c}^{2}}{J_{a}^{2} \zeta_{a}^{2}+4 J_{a c}^{2} \zeta_{a c}^{2}}
$$

which resolves to

$$
\frac{\zeta_{a c}^{2}}{\zeta_{a}^{2}}=\frac{J_{a}^{2}}{J_{a c}^{2}} \frac{2\left(J_{a c}-J_{a}\right)}{\left(2 J_{a}-J_{a c}\right)}\left(1-\frac{8\left(J_{a c}-J_{a}\right)}{\left(2 J_{a}-J_{a c}\right)}\right)^{-1} .
$$

The last relation implies that not only an upper boundary in $J_{a}$ for the Q1D-RVB state, but also a lower boundary may exist $\left(2 J_{a}>J_{a c}\right)$, although it may be not exactly determined from the above relation. The "control parameter" stipulating the change of the behavior here is $J_{a c}-J_{a}(>0)$ 
- the "depth" of penetration in the region where the Q1D-RVB state can exist. It in a way is a measure of frustration as well.

Pseudogapped 2D-RVB states. The spectrum for the pseudogapped 2D-RVB state with $\zeta_{a}, \zeta_{c} \neq 0, \zeta_{a c}=0$ unexpectedly integrates (with use of Mathematica $($ ). Indeed, we need to find

$$
-\frac{4}{\pi^{2}} \int_{0}^{\frac{\pi}{2}} \int_{0}^{\frac{\pi}{2}} \sqrt{A^{2} \cos ^{2} x+C^{2} \cos ^{2} z} d x d z
$$

where $A=3 J_{a} \zeta_{a}, C=3 J_{c} \zeta_{c}$. Performing first the integration with respect to $x$ we get

$$
-\frac{4 C}{\pi^{2}} \int_{0}^{\frac{\pi}{2}} \cos z \mathrm{E}\left(i \sqrt{\frac{A^{2}}{C^{2} \cos ^{2} z}}\right) d z .
$$

Second integration performs as well and yields

$$
\frac{C}{2 \pi^{3 / 2}} \mathbf{G}_{3,3}^{2,2}\left(\left.\frac{A^{2}}{C^{2}}\right|^{\frac{1}{2}, \frac{3}{2}, \frac{3}{2}} \begin{array}{l}
0,1,0
\end{array}\right),
$$

where a Meijer G-function enters into play. The result looks out unsymmetrically, however, one can prove applying formulae PBM III.8.2.2.14-15* that

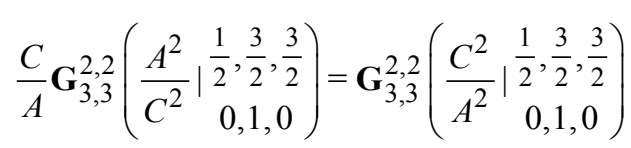

which shows as expected that the order of integration is indeed insignificant. Taking derivatives of the first representation of the kinetic energy with respect to $A$ and of the second one with respect to $C$, employing the chain rule, and combining them with the respective derivatives of the potential energy we arrive to the self-consistency equations for the OPs:

$$
\begin{aligned}
& \zeta_{c}=\frac{1}{2 \pi^{3 / 2}} \frac{J_{a}}{J_{c}} \mathbf{G}_{3,3}^{2,2}\left(\left(\frac{J_{a} \zeta_{a}}{J_{c} \zeta_{c}}\right)^{2} \mid \begin{array}{c}
2 \\
0,0,-1
\end{array}, \frac{1}{2}\right), \\
& \zeta_{a}=\frac{1}{2 \pi^{3 / 2}} \frac{J_{c}}{J_{a}} \mathbf{G}_{3,3}^{2,2}\left(\left(\frac{J_{c} \zeta_{c}}{J_{a} \zeta_{a}}\right)^{2} \mid \begin{array}{c}
-\frac{1}{2}, \frac{1}{2}, \frac{1}{2} \\
0,0,-1
\end{array}\right),
\end{aligned}
$$

where both OPs are assumed to be nonvanishing. The Meijer G-function has logarithmic nonanalyticities in the vicinities of the points $z=0, \infty$. Thus in the lowest order the expansions (observe the inverted arguments of the Gfunctions in Eq. (30))

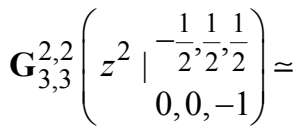

$$
\begin{aligned}
& \simeq \frac{\sqrt{\pi}}{2}\left(2 \ln \frac{8}{z}-1\right)+\frac{\sqrt{\pi}}{64}\left(19+12 \ln \frac{z}{8}\right) z^{2}+\ldots, \\
& \mathbf{G}_{3,3}^{2,2}\left(\frac{1}{z^{2} \mid} \begin{array}{c}
-\frac{1}{2}, \frac{1}{2}, \frac{1}{2} \\
0,0,-1
\end{array}\right) \simeq 2 \sqrt{\pi} z+\frac{\sqrt{\pi}}{2}\left(1+\ln \frac{z}{8}\right) z^{3}+\ldots
\end{aligned}
$$

both valid for $z \approx 0$ are used.

Assuming, according to the physical estimate, that a 1D-RVB state first installs along the $c$ direction we explore the limit

$$
z=\frac{J_{a} \zeta_{a}}{J_{c} \zeta_{c}} \ll 1
$$

Then using the lowest order estimates for the Meijer functions we get from the 2 nd equation

$$
\zeta_{c}=1 / \pi
$$

precisely as it used to be in the gapless 1D-RVB state. Inserting this in the 1st equation and using the lowest order estimate for the Meijer G-function we get

$$
\zeta_{a}=\frac{8}{\pi \sqrt{e}} \frac{J_{c}}{J_{a}} \exp \left(-\frac{2 J_{c}}{J_{a}}\right) .
$$

This shows that at arbitrary small ratio $J_{a} / J_{c}$ a self-consistent solution with two pseudogaps exist. Inserting this estimate back to the equation for $\zeta_{c}$ and using in the expansion of the Meijer G-function terms up to $z^{2}$ we get

$$
\zeta_{c}=\frac{1}{\pi}\left(1-\frac{1}{e} \exp \left(-\frac{4 J_{c}}{J_{a}}\right)\left[12-\frac{13 J_{a}}{2 J_{c}}\right]\right) .
$$

The latter shows that the correction to the OP $\zeta_{c}$ is exponentially small as one could expect.

Unfortunately, analogous moves did not bring success when it goes about more general expressions involving three nonvanishing OPs. For that reason we address this 2D-RVB state separately by other method (see below).

\section{Ground state of the $c-a-c a-R V B$ model}

In the previous section we obtained estimates of the self-consistent values of OPs for all nontrivial phases of the $c-a-c a$ model except one in relevant orders. This is, however, only a prerequisite for constructing the groundstate parameter phase diagram since at this point it is not known yet which of the self-consistent solutions corresponds to the lowest energy. In order to calculate the equilibrium energies of various RVB states we remind that by definition the "kinetic" energy at the zero temperature is a

* Hereinafter PBM r.a.a.a.a stands for the formula a.a.a.a of volume $r$ of Ref. 21. 
uniform function of the power one with respect to the OP's $\zeta_{a}, \zeta_{c}, \zeta_{a c}$ whereas the potential energy is the uniform function of the power two with respect to the same OP's. These are the conditions of the virial theorem. Thus for arbitrary positive numbers $z_{a}^{*}, z_{c}^{*}, z_{a c}^{*}$ defining a ray in the OP space the OPs are given by the scaling parameter $\lambda$ so that $\zeta_{\tau}=\lambda z_{\tau}^{*}$ and we get

$$
E\left(\lambda ; z_{\tau}^{*}\right)=\lambda T\left(z_{\tau}^{*}\right)+\lambda^{2} U\left(z_{\tau}^{*}\right)
$$

Optimizing with respect to $\lambda$ one obtains

$$
\lambda^{*}\left(z_{\tau}^{*}\right)=-\frac{T\left(z_{\tau}^{*}\right)}{2 U\left(z_{\tau}^{*}\right)}
$$

the equilibrium value of the scaling parameter characteristic for the chosen ray. The energy minimum along the ray $z_{a}^{*}, z_{c}^{*}, z_{a c}^{*}$ reads

$$
-\frac{T^{2}\left(z_{\tau}^{*}\right)}{2 U\left(z_{\tau}^{*}\right)}+\frac{T^{2}\left(z_{\tau}^{*}\right)}{4 U^{2}\left(z_{\tau}^{*}\right)} U\left(z_{\tau}^{*}\right)=-\frac{T^{2}\left(z_{\tau}^{*}\right)}{4 U^{2}\left(z_{\tau}^{*}\right)} U\left(z_{\tau}^{*}\right)=-U\left(\lambda^{*}\left(z_{\tau}^{*}\right) z_{\tau}^{*}\right)
$$

the last equality expressing the virial theorem for the given ratio of the powers of the uniform contributions (the total equilibrium energy equals minus potential energy calculated in the equilibrium point). Thus the total ground-state energy equals to minus potential energy calculated for the equilibrium values of the OP's which are known from the previous section. Thus for the states with only one nonvanishing OP we immediately get the following total energies:

$$
\begin{array}{ccc}
\text { 1D-RVB } & \text { 1D-RVB } & \text { 2D-RVB } \\
\zeta_{a}=\frac{1}{\pi} & \zeta_{c}=\frac{1}{\pi} & \zeta_{a c}=\frac{2}{\pi^{2}} \\
-\frac{3 J_{a}}{\pi^{2}} & -\frac{3 J_{c}}{\pi^{2}} & -\frac{24 J_{a c}}{\pi^{4}}
\end{array}
$$

The above states with one nonvanishing OP can be, however, unstable with respect to splitting of other OPs from zero values. For example, starting from the pseudogapless 2D-RVB state with only nonvanishing OP $\zeta_{a c}$ we obtain for the energy of the Q1D-RVB state opening a pseudogap with small $J_{a}$. The corresponding Q1D-RVB state with $\zeta_{a} \neq 0$ has the energy

$$
-6 J_{a c} \zeta_{a c}^{2}-3 J_{a} \zeta_{a}^{2}
$$

where the self-consistent values of the OPs have to be inserted. Taking those given by Eqs. (26), (27) we obtain for this quantity

$$
-\frac{24 J_{a c}}{\pi^{4}}-\frac{12 J_{a c}}{\pi^{4}} \cdot 16 \exp \left(-\frac{4 J_{a c}}{J_{a}}\right)
$$

which shows that the energy gain due to opening of the pseudogap is always larger by absolute value than the loss due to depletion of the OP $\zeta_{a c}$ which results in an overall energy gain although exponentially small. This result $m u$ tatis mutandis holds for $J_{c}$ as well which means that the gapless 2D-RVB state is always unstable for arbitrary small values of $J_{\tau} ; \tau=a, c$ and at zero temperature it decays to a Q1D-RVB state with $\zeta_{\tau} \neq 0$ whichever of $J_{\tau}$ is stronger. This is in certain correspondence with the results obtained in the high-temperature approximation $[11,12]$ which consistently show a decrease of the area in the parameters space where the gapless 2D-RVB phase exists with temperature decrease (see below).

For analysis of the 1D-RVB states we analogously use the potential energy of the 2D-RVB state:

$$
-3 J_{c} \zeta_{c}^{2}-3 J_{a} \zeta_{a}^{2}
$$

where the self-consistent values of the OPs have to be inserted. Using those given by Eqs. (31), (32) we get for the energy up to the lowest exponential terms

$$
-\frac{3 J_{c}}{\pi^{2}}-\frac{3}{\pi^{2} J_{a}} \exp \left(-\frac{4 J_{c}}{J_{a}}\right) \frac{1}{e}\left[64 J_{c}^{2}-24 J_{c} J_{a}+13 J_{a}^{2}\right] .
$$

The quadratic form in the square brackets is positive definite thus the energy correction is always negative and opening the missing pseudogap in any of the 1D-RVB states is energetically preferable thus leading to the pseudogapped 2D-RVB state.

As we noticed above the other boundary of a 1D-RVB phase, namely one with the Q1D-RVB one is of interest. Taking the expression (29) describing the ray on which the self-consistent values of the OPs characteristic of the Q1DRVB state must lay in the lowest order with respect to the control parameter $J_{a c}-J_{a}$ we derive for the Q1D-RVB ground-state energy:

$$
-\frac{3 J_{a}}{\pi^{2}}-\frac{12 J_{a}}{\pi^{2} J_{a c}} \frac{\left(J_{a c}-J_{a}\right)^{2}}{2 J_{a}-J_{a c}}
$$

which shows the quadratic gain due to transition from the 1D-RVB state to the corresponding Q1D-RVB state. This gain is obviously due to the shift of the logarithmic van Hove singularity towards lower energy as compared to the position of the power singularity of the $\mathrm{qDoS}$ in the strictly 1D-RVB state. Formally, the sign of the quadratic contribution changes at $2 J_{a}=J_{a c}$. It is not clear, however, for the moment whether the underlying expansions used so far remain valid in this parameter region.

So far we could establish that the (pseudo)gapless 1Dand 2D-RVB states are always unstable towards transitions leading either to Q1D- or pseudogapped 2D-RVB states. One can also suspect that the Q1D-RVB states are unstable with respect to opening of the pseudogap and going to the 2D-RVB state with three nonvanishing OPs. From the analysis of the high-temperature expansion it seems [11,12], however, that the 2D-RVB state with only two nonvanishing OPs squeezes out all other states and is the ground 
RVB state of the $c-a-c a$ model. In this situation it is important to check the stability of the pseudogapped 2D-RVB state with $\zeta_{a}, \zeta_{c} \neq 0, \zeta_{a c}=0$ with respect to widening of the bandwidth which is going to happen if the OP $\zeta_{a c}$ splits from zero. In order to calculate the kinetic energy one has to integrate

$$
-\frac{4}{\pi^{2}} \int_{0}^{\frac{\pi}{2}} \int_{0}^{\frac{\pi}{2}} \sqrt{A^{2} \cos ^{2} x+C^{2} \cos ^{2} z+4 B^{2} \cos ^{2} x \cos ^{2} z} d x d z
$$

where $B=3 J_{a c} \zeta_{a c}$. Performing first the integration with respect to $x$ we get

$$
-\frac{4 C}{\pi^{2}} \int_{0}^{\frac{\pi}{2}} \cos z \mathrm{E}\left(i \sqrt{\frac{A^{2}+4 B^{2} \cos ^{2} z}{C^{2} \cos ^{2} z}}\right) d z .
$$

The latter integrand is then expanded in assumption of a smallness of $B$ which yields the correction to the kinetic energy due to the bond widening:

$$
-\frac{8 B^{2}}{\pi^{2} C} \int_{0}^{\frac{\pi}{2}} \cos z \mathrm{D}\left(i \sqrt{\frac{A^{2}}{C^{2} \cos ^{2} z}}\right) d z
$$

Inserting the defininitions we get for the $\zeta_{a c}$-dependent contribution to the energy of the 2D-RVB state the following:

$$
-\frac{8 \cdot 9 J_{a c}^{2} \zeta_{a c}^{2}}{\pi^{2} C} I+6 J_{a c} \zeta_{a c}^{2},
$$

where $I$ is the above integral. This contribution is negative (the state with two nonvanishing OPs is unstable) provided

$$
\frac{12 J_{a c}}{\pi^{2} C} I>1 .
$$

With use of the formula PBM III.8.4.40.50 we obtain

$$
I=\frac{1}{2} \int_{0}^{\frac{\pi}{2}} \cos z \mathbf{G}_{2,2}^{2,1}\left(\left.\frac{C^{2} \cos ^{2} z}{A^{2}}\right|_{\frac{1}{2}, \frac{3}{2}} ^{1,2}\right) d z
$$

which after replacing the integration variable reduces to the integral

$$
I=\frac{1}{4} \int_{0}^{\frac{\pi}{2}} \frac{d y}{\sqrt{1-y}} \mathbf{G}_{2,2}^{2,1}\left(\left.\frac{C^{2} y}{A^{2}}\right|_{\frac{1}{2}, \frac{3}{2}} ^{1,2}\right)
$$

and with use of PBM III.2.24.2.2 yields

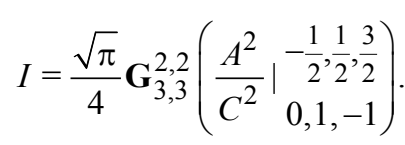

In the limit of small $A$ in the lowest order it results in the condition

$$
J_{a c}>J_{c}
$$

The latter estimate, however, does not take into account possible variation of the pseudogap-related OPs $\zeta_{a}, \zeta_{c}$ upon widening of the quasiparticle band. In order to take these latter into account we complement the self-consistency equations (30) by the terms proportional to $B^{2}$ stemming from the expansion of the integral (33). They basically reduce to taking derivatives of the above integral $I$ with respect to $A$ and $C$ and applying the chain rule. After doing that we see that the solution (32) of the equation for $\zeta_{c}$ in the state with three nonvanishing OPs acquires the form

$$
\frac{1}{\pi}-2 \pi\left(\frac{J_{a c} \zeta_{a c}}{J_{c}}\right)^{2}
$$

This results in a loss of the kinetic energy so that the overall lowest order contribution to the energy reads

$$
6\left(\frac{J_{a c}^{2}}{J_{c}}+J_{a c}\right) \zeta_{a c}^{2}
$$

which shows that the increasing the quasiparticle bandwidth in the psedogapped 2D-RVB state due to splitting the OP $\zeta_{a c}$ from zero increases the energy quadratically in this OP for all parameters' values, so that the 2D-RVB state with $\zeta_{a c}=0$ is stable: i.e., always has a lower energy than the state with $\zeta_{a c} \neq 0$.

The analysis performed in the present section shows that the extremely rich phase diagram of the $c$ - $a$ - $c a$ model existing at nonzero temperature completely degenerates at the zero temperature. All possible phases with lines of nodes in their respective quasiparticle spectra, i.e., the $1 \mathrm{D}$-, Q1D-, and pseudogapless 2D-RVB states turn out to be unstable with respect to opening of the missing pseudogaps. On the other hand the 2D-RVB state with vanishing $\zeta_{a c}$ OP is stable with respect to the splitting of this latter from the zero which makes us think that the RVB ground state of the $c-a-c a$ model is precisely that state. The most astonishing fact is that the same conclusion fairly follows from the high-temperature expansion [12]. Indeed, the cross-sections of the temperature-parameters phase diagram [12] shown in Fig. 4 demonstrate that up on the temperature decrease all phases are squeezed out by the 2D-RVB one with vanishing OP $\zeta_{a c}$ (depicted as orange), which according to our current study is the ground state of the model. This latter result confirms our observation that for the values of the reduced exchange parameters (baricentric coordinates in the parameters' space $J_{a}^{*}=0.172$; $\left.J_{c}^{*}=0.400 ; J_{a c}^{*}=1-J_{a}^{*}-J_{c}^{*}\right)$ characteristic for the CuNCN material (marked by a white star), i.e., within the Q1D-RVB phase (magenta), but close and somewhat below the quad- 


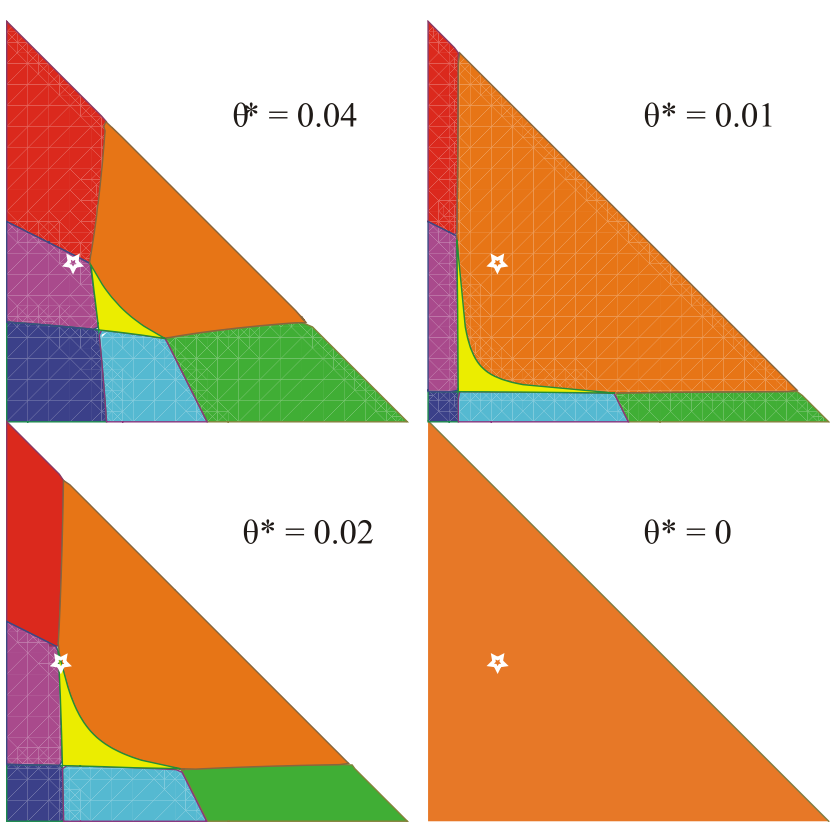

Fig. 4. (Color online) Parameter phase diagrams for the $c-a-c a$ RVB model in the high-temperature approximation. The abscissa and ordinate represent the reduced parameters $J_{a}^{*}$ and $J_{c}^{*}$; these parameters, respectively, equal to unity in the lower right and upper left corners; the entire set of reduced parameters is subject to the condition $J_{a}^{*}+J_{c}^{*}+J_{a c}^{*}=1$. The reduced temperatures $\theta^{*}$ are fractions of $J_{a}^{*}+J_{c}^{*}+J_{a c}^{*}$. The color coding for phases is: 1D-RVB with only one nonvanishing OP = red or green, gapless 2D-RVB = blue; Q1D-RVB = magenta, cyan; pseudogapped $2 \mathrm{D}-\mathrm{RVB}=$ orange for $\zeta_{a c}=0$; yellow codes the pseudogapped 2D-RVB phase with $\zeta_{a c} \neq 0$. The small star shows the tentative position of $\mathrm{CuNCN}$ on the parameter phase diagram.

ruple point of the red, magenta, orange, and yellow phases in the graph in the left upper corner $\left(\theta^{*}=0.04\right)$ the following sequence of transitions between the RVB phases:

$$
\begin{array}{ccc}
\text { Q1D-RVB } & \rightarrow \text { 2D-RVB } & \rightarrow \text { D-RVB } \\
\zeta_{c}, \zeta_{a c} & \rightarrow \zeta_{c}, \zeta_{a c}, \zeta_{a} & \rightarrow \zeta_{c}, \zeta_{a} \\
\text { magenta } & \rightarrow \text { yellow } & \rightarrow \text { orange }
\end{array}
$$

occurs up on decreasing temperature showing that the 2DRVB phase with three nonvanishing OPs (yellow) is a transient one and it is replaced by the 2D-RVB phase with two nonvanishing OPs (orange). Our current study confirms this conclusion by establishing that the pseudogapped 2D-RVB state (orange) phase is the RVB ground state. Of course, it does not exclude that for certain parameters' regions the RVB in general is not the ground state which is, of course, the case either for $J_{a c} \ll J_{a}, J_{c}$ or $J_{a c} \gg J_{a}, J_{c}$ when the ordered phases must occur. Here we, however, focus on the RVB states.

\section{Conclusion}

Recently we proposed that the fascinating physics of copper carbodiimide $(\mathrm{CuNCN})$ can be explained if one assumes a resonating valence bond (RVB) character of its phases. Such phases are expected to appear in two-dimensional Heisenberg models with frustrated antiferromagnetic couplings. Two models of this type are considered: a well known anisotropic triangular lattice model and a new spatially anisotropic antiferromagnetic Heisenberg model with the exchange parameters $J_{c}, J_{a}$, and $J_{a c}$ extending along the $c, a$, and $a \pm c$ directions of a two-dimensional rectangular lattice. The detailed analysis of the mean-field selfconsistency equations for RVB ground states yields the solutions for the order parameters. The relative positions of these solutions on the energy scale are determined. The ground state of the anisotropic triangular Heisenberg lattice model is always gapped at arbitrary weak frustrating coupling $J_{a b}$, although the magnitude of the gap is exponentially small for small $J_{a b}$. For the $c-a$ - $c a$ model having extremely rich temperature-parameters phase diagram we establish that the 2D-RVB state with two pseudogaps and the vanishing RVB order parameter coupled with the interaction along the structural diagonal $\left(\zeta_{a c}\right)$ is the only possible RVB ground state for all values of parameters. The latter finding is in a fair agreement with our high-temperature analysis implying the transient character of the phase with three nonvanishing OPs.

\section{Acknowledgments}

This work has been performed with the support of DFG. Discussion with Prof. V.Ya. Krivnov and Dr. D. Dmitriev is acknowledged.

1. X.-H. Liu, R. Dronskowski, R.K. Kremer, M. Ahrens, C.-D. Lee, and M.-H. Whangbo, J. Phys. Chem. C 112, 11013 (2008).

2. H. Xiang, X.-H. Liu, and R. Dronskowski, J. Phys. Chem. C 113, 18891 (2009).

3. A.L. Tchougréeff and R. Dronskowski, arXiv:1008.0182.

4. A. Zorko, P. Jeglič, A. Potočnik, D. Arčon, A. Balčytis, X. Liu, A.L. Tchougréeff, and R. Dronskowski, Phys. Rev. Lett. 107, 047208 (2011).

5. A.L. Tchougréeff and R. Dronskowski, arXiv:1111.7210.

6. A.L. Tchougréeff, X. Liu, P. Müller, W. van Beek, U. Ruschewitz, and R. Dronskowski, J. Phys. Chem. Lett. 3, 3360 (2012).

7. M. Ogata and H. Fukuyama, Rep. Progr. Phys. 71, 036501 (2008).

8. P.W. Anderson, Mater. Res. Bull. 8, 153 (1973).

9. L. Balents, Nature 464, 199 (2010).

10. J.B. Goodenough, Magnetism and the Chemical Bond, Interscience-Wiley, New York (1963).

11. A.L. Tchougréeff and R. Dronskowski, arXiv:1304.2596. 
12. A.L. Tchougréeff and R. Dronskowski, J. Phys.: Condens. Matter 25, 435602 (2013).

13. M. Ogata, J. Phys. Soc. Jpn. 72, 1839 (2003).

14. Janke-Emde-Lösch, Tafeln Höhere Funktionen. 6. Auflage Neubearbeitet von F. Lösch, B.G. Teubner Verlagsgeselschaft, Stuttgart (1960).

15. P. Fulde, Electron Correlations in Molecules and Solids, 2nd edition, Springer-Verlag, Berlin (1993).

16. Y. Hayashi and M. Ogata. J. Phys., Conf. Ser. 150, 042053 (2009).

17. A.E. Belinskii, A.L. Tchougréeff, and I.A. Misurkin, Teor. Eksp. Khim. 25, 513 (1989) [Theor. Exp. Chem. 25, 475 (1989)].

18. Y. Hayashi and M. Ogata, arXiv:0704.1313v1; Y. Hayashi and M. Ogata, J. Phys. Soc. Jpn. 76, 053705 (2007).
19. A.A. Nersesyan and A.M. Tsvelik. Phys. Rev. B 67, 024422 (2003).

20. P. Hauke, T. Roscilde, V. Murg, J.I. Cirac, and R. Schmied, New J. Phys. 13, 075017 (2011); M. Kohno, L. Balents, and O.A. Starykh, J. Phys.: Conf. Ser. 145, 012062 (2009); P. Chandra and B. Doucot, Phys. Rev. B 38, 9335 (1988); M. Mambrini, A. Läuchli, D. Poilblanc, and F. Mila, Phys. Rev. $B$ 74, 144422 (2006); F. Figueirido, A. Karlhede, S. Kivelson, S. Sondhi, M. Rochek, and D.S. Rokhsar, Phys. Rev. B 41, 4619 (1989); N. Read and S. Sachdev, Phys. Rev. Lett. 66, 1773 (1991); J. Ferrer, Phys. Rev. B 47, 8769 (1993).

21. A.P. Prudnikov, Yu.A. Brychkov, and O.I. Marichev, Integrals and Series, vv. I-V, Gordon and Breach Science Publishers, New York (1986-1992). 